\title{
Risk Factor and Diagnosis of Sacral Insufficient Fracture after Multilevel Fusion: A Case Report and Literature Review
}

\author{
Myungseok Lee, Dong Ho Kang ${ }^{凶}$, Young Suk Lee, Dong Hyun Chun, Ja Myeong Lee \\ Department of Neurosurgey, Gyeongsang National University College of Medicine, Jinju, Korea
}

\begin{abstract}
Sacral insufficiency fracture occurs with normal stress on abnormal bone. Sacral fractures after posterior multilevel lumbosacral fusion are uncommon complications. Only a few case series and case reports have been published so far. Here we describe five patients who have sacral fracture after multilevel fusion surgery. We retrospectively reviewed all patients treated at our institution by the author (Pf. K) for sacral fractures following an instrumented spinal arthrodesis with extension to S1 during the period of 2008 to 2015 (Total 158 cases). We describe these five patients' characteristics, risk factors, symptom after surgery, and treatment plan. There was delay in diagnosis because it was difficult to find it in plain radiography. Computed tomography and MRI can detect most such fractures. Thus, they should probably be performed routinely when patients complain of renewed buttock pain after lumbosacral fusion. There are mainly three optional treatments for sacral fracture: conservative treatment, sacroplasty, and revision operation. Three patients who underwent revision surgery explained that their back pain and leg pain were improved dramatically. Early surgery can be considered because it can help patients relieve pain and ambulation earlier. Even though treatment of choice for sacral fracture is conservative management, surgery should be considered as one of good options for sacral fracture.
\end{abstract}

Key Words: Sacral fracture; Insufficiency fracture; Surgical treatment; Complication; Multilevel lumbosacral fusion

$\triangle$ Corresponding Author: Dong Ho Kang, Department of Neurosurgery, Gyeongsang National University College of Medicine, 79, Gangnam-ro, Jinju 52727, Korea. Tel: +82-55-750-8112, Fax: +82-55-759-0817, E-mail: ns4793@hanmail.net

\section{INTRODUCTION}

Spinal instrumentation surgery has been an effective treatment option for patients with symptomatic degenerative disease. Postoperative complications are more common at the L5-S1 level than those at other levels in the thoracolumbar spine ${ }^{2}$. Although fusion surgery has several advantages, some complications have been reported. One major complication is adjacent segment failure. The rigidity of the fused segment influences abnormal stress on adjacent levels. Sacral fractures below instrumented fusion constructs are relatively rare sources of postoperative pain. Only a few cases have been previously reported in the literature ${ }^{1,3,4,-9)}$. Early sacral fracture is a rare complication of lumbosacral fusion. Because the majority of patient of spinal stenosis are the elderly, they have risk of osteoporosis that is major risk factor of sacral insufficiency fracture. Risk factors of sacral insufficiency fracture are old age, female, osteoporosis, use of chronic corticosteroids, multi-segmental fixation, mismatched LL-PI, and L5-S1 pseudoarthrosis ${ }^{10,12-15,17)}$. It is difficult to diagnose sacral fractures with routine plain $\mathrm{X}$-ray examination. This can lead to delayed or missed diagnosis. Because of its rarity, there is no protocol for diagnosis and treatment of sacral fractures. Here we present five cases of sacral fractures after lumbosacral fusion. With these five cases, we tried to review patient characteristics and discuss how to diagnose and treat these complications.

\section{ILLUSTRATIVE CASES}

\section{Case 1}

An 80-year-old woman complained two years of gradually increased low back pain, both buttock pain, bilateral leg pain, and tingling sense. From two years ago, she needed a walker for self-ambulation. From one year ago, she had voiding difficulty. Her previous medical history was significant for hypertension, diabetic mellitus (DM), stent insertion due to myocardiac infarction. X-ray showed spondylolisthesis, grade I, L3 on L4, MRI showed multiple spinal stenosis, L3/4/5/S1. In her long bone radiographs, we found that her PI value was $47.2^{\circ}$ and LL value was $8.2^{\circ}$ which makes PI-LL mismatch increased to $39^{\circ}$ (Fig. 1A). Physical examination demonstrated grade 3 motor weakness on ankle flexion and dorsiflexion with poor balance. She underwent L3/4/5/S1 total laminectomy, 
facetectomy, foraminotomy, and L3/4 interbody fusion with cage insertion and $\mathrm{L} 3 / 4 / 5 / \mathrm{S} 1$ pedicle screw insertion with posterolateral fusion. In operation room, cement augmentation was decided to supplement because of her osteoporotic weak bone (Fig. 1B). The patient's postoperative course was uneventful. Until 3 weeks after the first surgery, she underwent mild back and leg pain, but four weeks after the surgery, she had severe back pain and both anterior thigh pain. Pain aggravated gradually. Plain radiographs were not remarkable and magnetic resonance images (MRI) was done and revealed transverse S1 fracture (Fig. 1C) and she underwent a revision surgery with bilateral S2-alar-iliac screw extended fixation (Fig. 1D). As usual, thoracolumbosacral orthosis was used to immobilize the operation site. Five months after revision surgery, she complained mild left thigh pain, although other symptoms were relieved. Serial plain X-ray showed that S1 fracture was well fused. The patient had only mild pelvic pain. After eleven months postoperatively, she was walking quite well.

\section{Case 2}

The second case was a 59-year-old man who presented with a 2-year history of back pain and left lower extremity pain that continued from buttock to toe. He also had numbness and severe tingling sensation while he was walking. His medical history included uncontrolled DM and hypertension. In physical examination, there was no motor weakness. Radiographs revealed grade II of L5/S1 spondylolytic spondylolisthesis with severe foraminal stenosis from L5 to S1. In his long bone radiographs, we found that his pelvic incidence was $70^{\circ}$ (Fig. $2 \mathrm{~A}, \mathrm{~B})$. He underwent laminectomy and foraminotomy at L4/ $5 / \mathrm{S} 1$ level, discectomy at $\mathrm{L} 5 / \mathrm{S} 1$, and pedicle screw and rod fixation at L4-5-S1 (Fig. 2C). His immediate postoperative course was favorable. Symptom was relieved. He ambulated
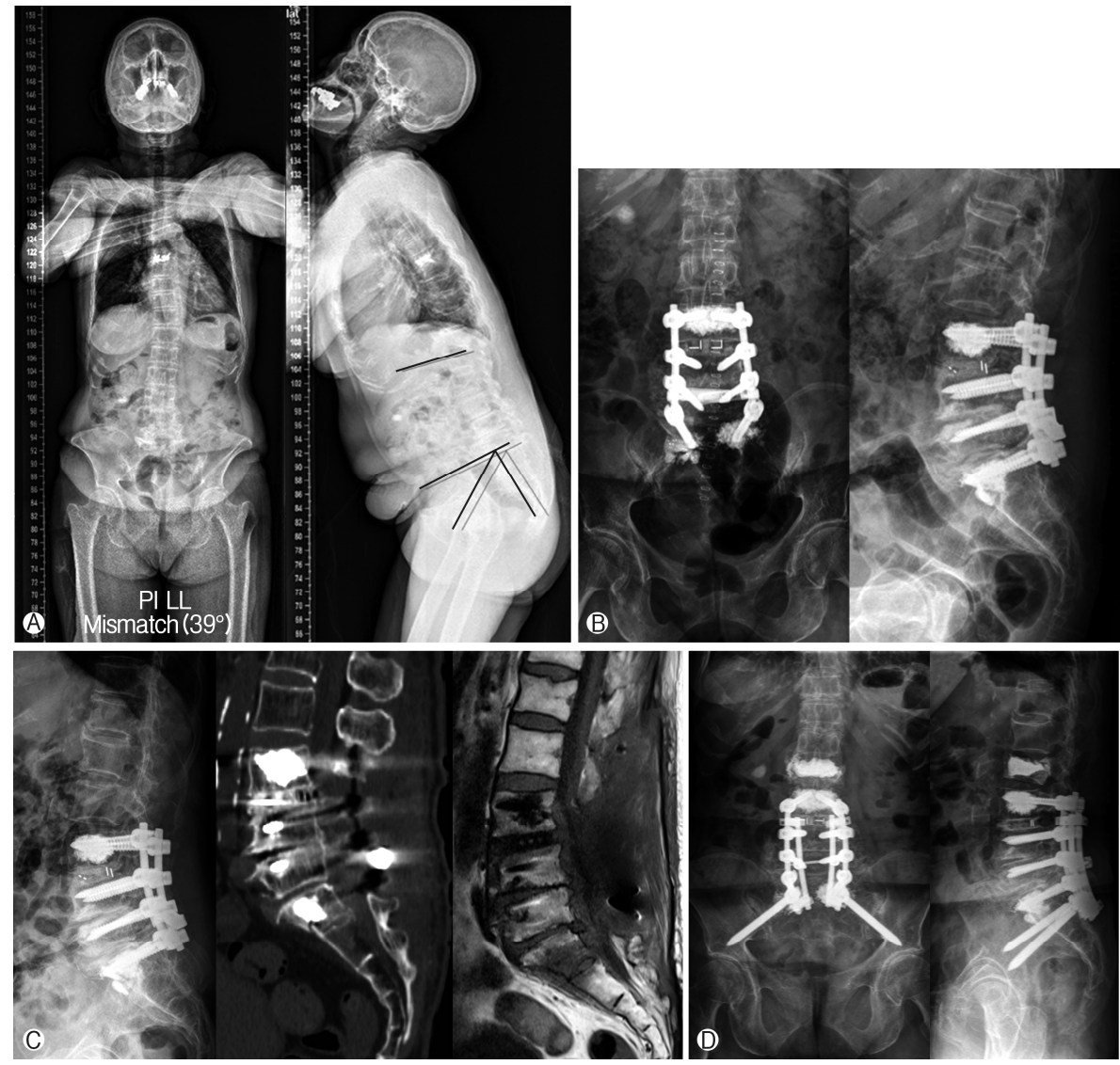

Fig. 1. Case. 1. Pre-operation X-ray finding. Her PI-LL mismatch was increased to $39^{\circ}$. Preoperation MRI finding showed mild spondylolisthesis, grade I, L3 on L4, Spinal stenosis, L34-5-S1 (A). X-ray findings after $1^{\text {st }}$ operation (B). After 4 weeks later, fracture on S1 body was not detected on X-ray and CT and MRI reveal sacral fracture. Under the S1 rod, fracture line can be found in CT scan. At the same lesion, there is an acute vertebral body compression fracture of S1. Decreased signal intensity within this vertebral body on the T1-weighted scan (C). X-ray findings after second operation, she underwent a revision surgery with bilateral S2alar-iliac screw fixation (D). 
well without pain. At 4 weeks later, he visited our emergency room with severe back pain and radiating pain and numbness on the entire left leg. He was unable to walk for 5 minutes. Plain radiographs could not clearly demonstrate a transverse sacral fracture. Therefore, we performed computed tomography (CT) and MRI scan for diagnosis. Results revealed a sacral proximal plate fracture with S1 screws failure (Fig. 2D). He underwent a revision surgery with extension of both double iliac screw insertion and rod fixation (Fig. 2E). The patient's back and lower extremity pain was improved dramatically over the course of two weeks after surgery. The patient made a satisfactory recovery with only little sacral pain that was not affecting daily activities.

\section{DISCUSSION}

Spinal instrumentation and fusion for degenerative diseases in the lumbar spine have been shown to have excellent fusion rates $^{16,18)}$. Sacral fractures after posterior lumbosacral fusion are uncommon. Although there are some published case reports, the number of cases is small in number ${ }^{1,3,4,6-9)}$. Biomechanical studies have demonstrated that load distributions are altered with long fusion constructs. However, the rigidity of long segmental instrumentation places significant stresses on adjacent spinal levels ${ }^{20)}$.

Because of their rare incidence, majority of spine surgeons do not have chance to face these fractures. Otherwise, even though there were fractures, surgeons might fail to detect these fractures. This is because most of these fractures are not easy to detect on plain radiographs. Therefore, sacral fractures could have been underestimated. Their diagnosis should be performed by CT or MRI or bone scan. As we can see from our cases, sacral fractures after lumbosacral fusion could have been largely underdiagnosed. It has been proven that CT can detect most of such fractures. Therefore, it should probably be performed routinely when patients complain of renewed buttock pain after lumbosacral fusion without definite radiographic abnormalities. Insufficiency fractures are insidious in onset. They usually present with poor localization and vague complaints of secondary

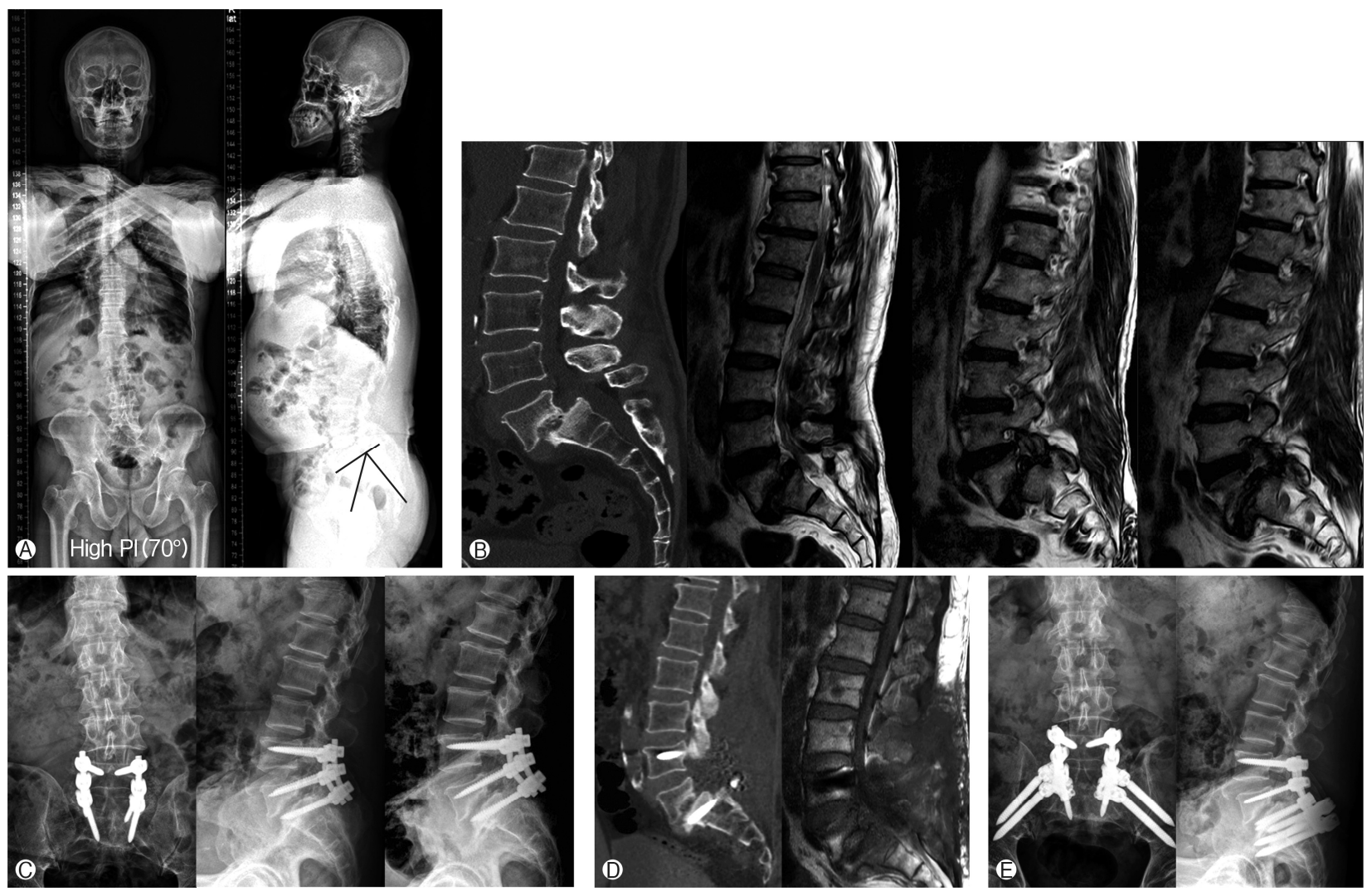

Fig. 2. Case 2. Pre-operation $x$-ray finding. We found that his pelvic incidence was highly increased $\left(70^{\circ}\right)$. Pre-operation $M R I$ finding revealed grade II spondylolisthesis and L5/S1 lumbar spondylosis with severe foraminal stenosis from L5 to S1 (A) and (B). X-ray finding after first operation. L4/5/S1 level, discectomy at L5/S1, and pedicle screw and rod fixation at L4-5-S1 (C). Under the S1 rod, prominent S1 body fracture can be found in CT scan. At the same lesion, there is an acute vertebral body compression fracture of S1. Decreased signal intensity within this vertebral body on the T1-weighted scan (D). X-ray finding after second operation. He underwent a revision surgery with extension of both double iliac screw insertion and rod fixation (E). 
Table 1. Patient's demographics

\begin{tabular}{|c|c|c|c|c|c|c|c|c|c|c|c|}
\hline Case & Age & Sex & Diagnosis & $\begin{array}{c}\text { Fusion } \\
\text { level }\end{array}$ & $\begin{array}{c}\begin{array}{c}\text { Pain onset } \\
\text { (weeks) }\end{array} \\
\end{array}$ & Location & BMD & Comorbidity & Treatment & $\begin{array}{c}\text { preop } \\
\text { PI-LL }\left(^{\circ}\right) \\
\end{array}$ & $\begin{array}{l}\text { preop } \\
\text { PI }\left(\left(^{\circ}\right)\right. \\
\end{array}$ \\
\hline 1 & 80 & $\mathrm{~F}$ & $\begin{array}{l}\text { Spinal stenosis and sagittal } \\
\text { imbalance }\end{array}$ & L3-S1 (4) & 4 & S1 & -3.1 & $\mathrm{HT}, \mathrm{DM}$ & Iliac screw & 39 & 47.2 \\
\hline 2 & 59 & M & $\begin{array}{l}\text { Spondylolisthesis L5-S1, } \\
\text { Grade II }\end{array}$ & L4-S1 (3) & 4 & S1 & -1.1 & HT, DM & Iliac screw & 4.3 & 70 \\
\hline 3 & 79 & $\mathrm{~F}$ & Spinal stenosis & L2-S1 (5) & 15 & S1 & -3.7 & COPD & Iliac screw & UC & UC \\
\hline 4 & 83 & $\mathrm{~F}$ & Spinal stenosis & L4-S1 (3) & 6 & S1 & -3.3 & Spondylitis & Conservative & UC & UC \\
\hline 5 & 57 & $\mathrm{~F}$ & $\begin{array}{l}\text { Spondylitis, Adjacent segmental } \\
\text { degeneration, L5-S1 }\end{array}$ & L2-S1 (5) & 3 & S1 & -1.8 & $\begin{array}{l}\text { HT, DM, } \\
\text { ESRD }\end{array}$ & Expired & UC & UC \\
\hline
\end{tabular}

HT: hypertension, DM: Diabetic mellitus, COPD: Chronic obstructive pulmonary disease, ESRD: End-stage renal disease, BMD: Bone mineral density, PI-LL: Pelvic incidence-Lumbar lordosis, PI: Pelvic incidence, UC: Unchecked.

increase of low back pain. In our series, most of these fractures seem to originate around the sacral screws. The screw may itself act as a stress riser and predispose the sacrum to this type of fracture.

Prior to planning fusion, especially if sacrum level is involved, surgeons should consider risk factors of patients. Old age, female sex, osteoporosis, obesity, and multi-segmental lumbosacral fusion are well known risk factors for sacral fractures. Mismatched LL-PI and high PI are also risk factors ${ }^{12,14,17}$.

Our cases confirmed these results (Table 1). Between 2008 to 2015, our institute performed 158 surgery which fusion ended at S1 level. We experienced 5 cases of sacral fracture. The percentage is about $3 \%$, which is similar with previous reports. However, our study revealed another possible risk factor for these fractures. In case 2, even though the patient was a relative young male patient with normal BMD score, he was suffering from this fracture. We partially reduced spondylolisthesis through pulling L5 screw for indirect decompression during surgery. However, we were not able to insert cage because of not securing of enough space for cage. Therefore, we think that significant shearing forces would be concentrated on S1. The shearing forces to S1 would be more aggravated because load transmission will be almost going through instrument and S1 due to none of interbody fusion.

As we can see in these cases, without a high index of suspicion, sacral fractures can be easily underestimated which can cause diagnostic delay. With review of risk factors of sacral fracture, it can help surgeon predict and avoid this complication.

As for their treatment, there are three major treatment plans. Many studies have reported that these postoperative sacral fractures respond well to conservative treatments that include activity modification, external immobilization, and medical treatment of osteoporosis ${ }^{1,3,4,11)}$. However, sacral insufficiency fractures with significant displacement, sagittal imbalance, neurologic symptoms, or painful nonunion require surgical stabilization $^{1,20)}$. There are a variety of surgical procedures to enhance balance and stability.
The most commonly performed procedure is to extend the fusion construct to the iliac wings using iliac screws. Fracture union and pain relief are achieved in all surgically treated cases reported in the literature. This determination can be made based on clinical symptoms and fracture alignment.

In our study, lumbopelvic fixation is a reliable and effective method to restore alignment and displacement of these fractures, especially in old patient who have high risk of complications (such as pneumonia, PTE, and general weakness) through prolonged bed rest.

\section{CONCLUSION}

Sacral insufficiency fractures are uncommon complications of multilevel lumbosacral fusion in osteoporotic patients. Surgeons should suspect postoperative S1 fracture when patients undergone instrumented fusion to S1 complain newly developed pain or gradually increasing pain during postoperative course. If there are these symptoms, surgeons should take CT scan or MRI for exact diagnosis because the diagnosis may be difficult in plain radiography. Although majority of sacral fractures can be cured by conservative treatment, lumbopelvic fixation can be considered as useful treatment modality for such patients since most patients are old age and these patients will have high possibility of risk for other complications through prolonged bed rest.

\section{- CONFLICT OF INTEREST}

The authors declare that they have no proprietary, commercial, or financial interests that could be construed to have inappropriately influenced this study.

\section{- NOTES}

This study has not been presented in part elsewhere.

\section{REFERENCES}


1. Bose B: Fracture of S1-2 after L4-S1 decompression and fusion. J Neurosurg 99:310-312, 2003

2. Edwards CC 2nd, Bridwell KH, Patel A, Rinella AS, Berra A, Lenke LG: Long adult deformity fusions to L5 and the sacrum. A matched cohort analysis. Spine 29:1996-2005, 2004

3. Elias WJ, Shaffrey ME, Whitehill R: Sacral stress fracture following lumbosacral arthrodesis. Case illustration. J Neurosurg 96: 135,2002

4. Fourney DR, Prabhu SS, Cohen ZR, Gokaslan ZL, Rhines LD: Early sacral stress fracture after reduction of spondylolisthesis and lumbosacral fixation: case report. Neurosurgery 51:15071510,2002

5. Gotis-Graham I, McGuigan L, Diamond T, Portek I, Quinn R, Sturgess A, et al.: Sacral insufficiency fractures in the elderly. J Bone Joint Surg Br 76:882-886, 1994

6. Khan MH, Smith PN, Kang JD: Sacral insufficiency fractures following multilevel instrumented spinal fusion. Spine 30:E484488, 2005

7. Khanna AJ, Kebaish KM, Ozdemir HM, Cohen DB, Gonzales RA, Kostuik JP: Sacral insufficiency fracture surgically treated by fibular allograft. J Spinal Disord Tech 17:167-173, 2004

8. Klineberg E, McHenry T, Bellabarba C, Wagner T, Chapman J: Sacral insufficiency fractures caudal to instrumented posterior lumbosacral arthrodesis. Spine 33:1806-1811, 2008

9. Koh YD, Kim JO, Lee JJ: Stress fracture of the pelvic wing-sacrum after long-level lumbosacral fusion: a case report. Spine 30:E161163, 2005

10. Legaye J , Duval-Beaupere G, Hecquet J, Marty C: Pelvic incidence: a fundamental pelvic parameter for three-dimensional regulation of spinal sagittal curves. Eur Spine J 7:99-103, 1998

11. Mathews V, McCance SE, O'Leary PF: Early fracture of the sacrum or pelvis: an unusual complication after multilevel instrumented lumbosacral fusion. Spine 26:E571-575, 2001
12. Odate S, Shikata J, Kimura H, Soeda T: Sacral fracture after instrumented lum-bosacral fusion: analysis of risk factors from spinopelvic parameters. Spine 38(4):E223-229, 2013

13. Roussouly P, Gollogly S, Berthonnaud E, Dimnet J: Classification of the normal variation in the sagittal alignment of the human lumbar spine and pelvis in the standing position. Spine 30:346353, 2005

14. Scemama C, D'astorg H, Guigui P: Sacral stress fracture after lumbar and lumbosacral fusion. How to manage it? A proposition based on three cases and literature review. Orthop Traumatol Surg Res 102:261-268, 2016

15. Schwab F, Lafage V, Boyce R, Skalli W, Farcy JP: Gravity line analysis in adult volunteers: age-related correlation with spinal parameters, pelvic parameters, and foot position. Spine 31:E959967, 2006

16. Schwab FJ, Nazarian DG, Mahmud F, Michelsen CB: Effects of spinal instrumentation on fusion of the lumbosacral spine. Spine 20:2023-2028, 1995

17. Shono Y, Kaneda K, Abumi K, McAfee PC, Cunningham BW: Stability of posterior spinal instrumentation and its effects on adjacent motion segments in the lumbosacral spine. Spine 23(14): 1550-1558, 1998

18. Thomsen K, Christensen FB, Eiskjaer SP, Hansen ES, Fruensgaard $S$, Bünger CE: The effect of pedicle screw instrumentation on functional outcome and fusion rates in posterolateral lumbar spinal fusion: a prospective, randomized clinical study. Spine 22: 2813-2822, 1997

19. Wang Y, Liu XY, Li CD, Yi XD, Yu ZR: Surgical treatment of sacral fractures following lumbosacral arthrodesis: Case report and literature review. World J Orthop 7:69-73, 2016

20. Zaman FM, Frey M, Slipman CW: Sacral stress fractures. Curr Sports Med Rep 5:37-43, 2006 